\title{
Buckling of Thin Films in Nano-Scale
}

\author{
S.B. Wang ${ }^{1 \text { a }}$, H.K. Jia ${ }^{1}$, J.Q.Sun ${ }^{1}$, X.C. Ren ${ }^{1}$, L.A. Li $^{1}$ \\ ${ }^{1}$ Mechanics Department, Tianjin University, Tianjin, 300072, China
}

\begin{abstract}
Investigation of thin film buckling is important for life prediction of MEMS device which are damaged mainly by the delamination and buckling of thin films. In this paper, the mechanical and thermal properties of compressed thin film titanium films with $150 \mathrm{~nm}$ thickness deposited on an organic glass substrate under mechanical and thermal loads were measured and characterized. In order to simulate the thin films which subjected to compound loads and the buckle modes, the external uniaxial compression and thermal loading were subjected to the specimen by the symmetric loading device and the electrical film in this experiment. The temperature of the thin film deposited on substrate was measured using thermoelectric couple. The range of temperature accords with the temperature range of the MEMS. It is found that the size and number of the delamination and buckling of the film are depended upon the pre-fixed mechanical loading and thermal temperature. The thermal transient conduction and thermal stability of the film and substrate was studied with finite element method.
\end{abstract}

Keywords: thin film buckling, electrical foil, temperature, symmetric loading device, nano-scale

\section{Introduction}

Film/substrate structure in information science occupies an important position, for example, data storage and processing systems on integrated circuits contain a large number of conductive, semi-conductive and insulating films, the magnetic films which play a key role in the disk storage systems, etc.

\footnotetext{
a e-mail: shbwang@tju.edu.cn
} 
However, the above-mentioned thin film/substrate system withstand a variety of load at work ( such as cutting tools, anti-corrosion coating), thermal stress caused by the heat ( such as the micro-chip packaging coating), especially the residual stress in the film, either the thermal mismatch produced in the high-temperature deposition process and the subsequent cooling process, or the internal stress caused by lattice mismatch [1]. Because delamination and buckling is the main failure modes of these devices, so the investigation of buckling is of great significance for its life prediction.

Thin-film buckling (buckle) generally considered refers to a failure mode caused by the compressive force on the film material, characterized by the vertical displacement perpendicular to load direction. Buckling based on specific patterns can be divided into [2, 3]: the straight-sided wrinkle; the circular blister or bubble; the telephone cord buckle; The expansion model of straight-sided wrinkle is recognized in the steady-state expansion conditions that the wrinkle expands along the curved front of the oval; while the linear edge formatted subsequently do not expand (J. W. Hutchinson [2]). Also, he proposed the cross-section of straight-sided wrinkle shows the shape of cosine curve. It has been shown by M. W. Moon [4] that the telephone cord topology can be effectively modeled as a series of pinned circular buckles along its length, with an unpinned circular buckle at its front. A. Lee [5] believes that the advantages of this model lies in its curved edge, the calculation of the finite element model with internal stress close to experimental values [4] based on this understanding. J. P. Eymery [6] studied the bubble-like buckling of the stainless steel membrane deposited on the silicon wafer caused by the residual stress. The initial expansion maintains round, but with the increase of residual stress, the bubble mutated into lobe. Some circular buckles have small straight-sided wrinkles through them. Observations of a large number of circular buckling show that in the expansion process, the crack model of bubbles will change from type I into type II. As the load increased further, the straight-sided wrinkle will transform to varicose mode. Interpretation of M. W. Moon [7] is that the varicose mode is the transition model from the straight-sided wrinkle to the telephone cord buckle. With the stress increase of in buckling load the straight-sided wrinkle will finally change to the telephone cord buckle.

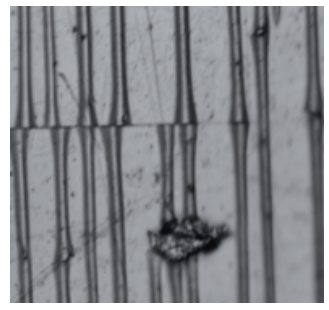

$21.8^{\circ} \mathrm{C}$

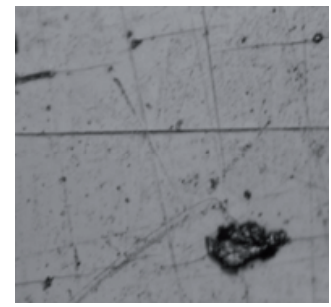

$54.9^{\circ} \mathrm{C}$

Fig.1 Buckling and expansion of the film as temperature increasing.

Wang, et al [8] investigated the mechanical and thermal properties of compressed thin film titanium films with 150nm thickness deposited on an organic glass substrate under mechanical and thermal 
loads. It is found that the straight-sided wrinkles produced by pre-fixed mechanical loading disappeared as the mechanical loading increasing, shown in Fig. 1.

\section{Measurement system}

An automatic control system of the displacement and force was developed, so as to apply precise remote force and displacement controlled by pc. Two PZT are used to apply deformation in this system. Automatic temperature control system was develop, which can apply thermal loading accurately. The mechanical and thermal properties of monolayer films were measured and characterized with different compression.

\subsection{Specimen}

In this paper, magnetron sputtering is used to produce the thin films specimen. The sample configuration is presented in Fig. 2 . The substrate dimensions are nominally $3 \times 9 \times 11 \mathrm{~mm} 3$. A $500 \mathrm{~nm}$ $\mathrm{Ti} / \mathrm{Ni}$ film was deposited on the organic substrate. The material of the substrate is organic glass.

Thin film is $500 \mathrm{~nm}$ in thickness. Electrical resistance film is used for applying thermal load.

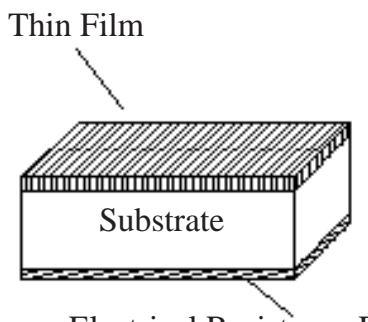

Electrical Resistance Film

Fig. 2 Configuration of the sample with Ti/Ni film.

\subsection{Symmetric loading device}

The experimental equipment is shown in Fig. 3. The specimen was deformed by two piezoelectric translators (PZT). During the compression, the displacements of the two sides of the specimen and applied load were controlled and recorded. A digital optical microscope (Olympus dp10) was placed above the films to observe the surface topography of the specimen (Fig. 2). The displacements of the two slide carriages are symmetric to achieve in situ observation in the view of microscope. 


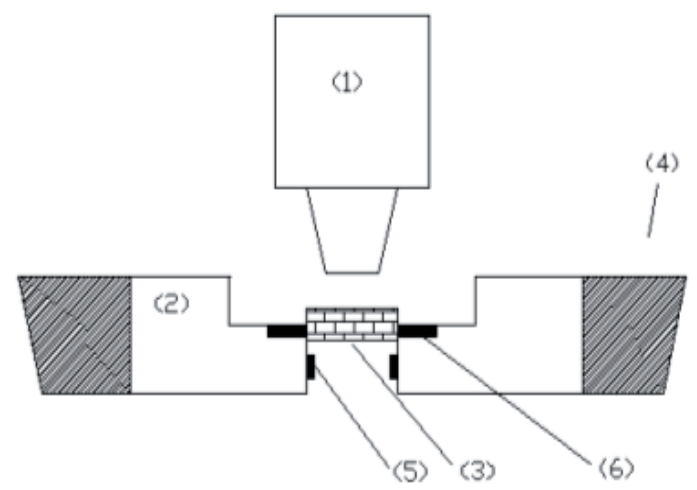

Fig. 3 The experimental equipment. (1) Olympus optical microscope dp10, (2) Slide carriages,

(3) Specimen, (4) PZT, (5) Displacement sensor, and (6) load sensor.

\subsection{Experimental results and discussion}

To measure and analyze the topology of thin-film under different pre-compression as the temperature changing, a number of group tests is carried out and recorded by a series of test images.

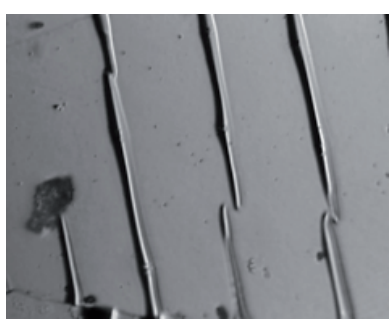

(a) $28^{\circ} \mathrm{C}$

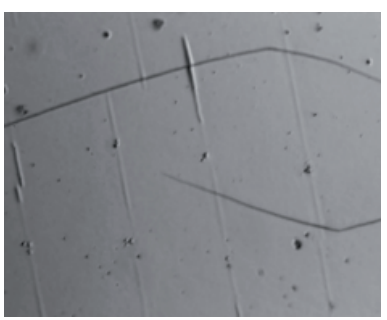

(b) $90^{\circ} \mathrm{C}$

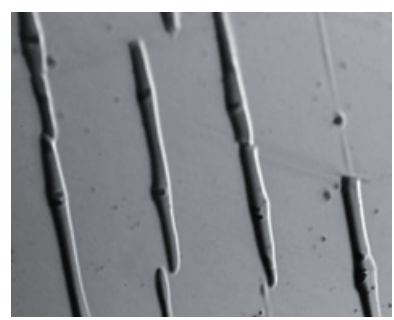

(c) $28^{\circ} \mathrm{C}$

Fig. 4 Buckling of the thin film with temperature changing, when axial load is $3.7 \mathrm{MPa}$.

Fig. 4 (a) shows the straight-sided topology of thin film produced by compression under room temperature. As shown in Fig. 4 (b), the resulting buckling gradually disappeared as the temperature increases, while the original small buckles completely disappear under the effect of the thermal load, the original larger buckles do not completely disappear under the thermal load. As the temperature lowered to room temperature, along the tracks of initial buckling produced by compression produced, thin film returned to the original straight-sided topology, and finally buckles down to room temperature, its width is wider than the width of initial buckling, as shown in Fig. 4. (c).

In Fig.5 (a), without thermal load, due to axial compression, film is result in straight-sided buckles with fractures across its. The fractures are caused by the different poison's ratio between the film and substrate. As the temperature increases further, straight-sided buckles decreases. In Fig. 5 (b), when the temperature rises to $90{ }^{\circ} \mathrm{C}$, the initial buckles leave only a few small local buckling. When the temperature dropped to the initial state, thin film returned to the original straight-sided topology with expansion at the front of buckles. 


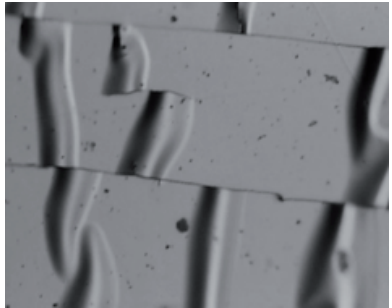

(a) $28^{\circ} \mathrm{C}$

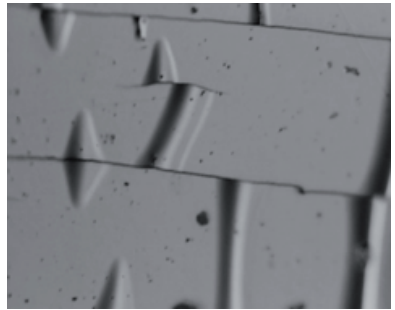

(b) $90^{\circ} \mathrm{C}$

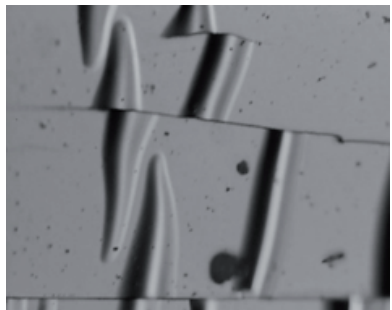

(c) $28^{\circ} \mathrm{C}$

Fig. 5 Buckling of the thin film with temperature changing, when axial load is $5.6 \mathrm{MPa}$.

\section{Finite element analysis}

The temperature measured in the experiment is the heating temperature at the bottom of the specimen. The finite element mode of the substrate was established to analyze the stress change under different compressions with temperature variations. First, the temperature field analysis of the substrate resulted by different heating temperatures. Then the temperature field is coupled with pre-stress field, to calculate the stress changes at the observation points.

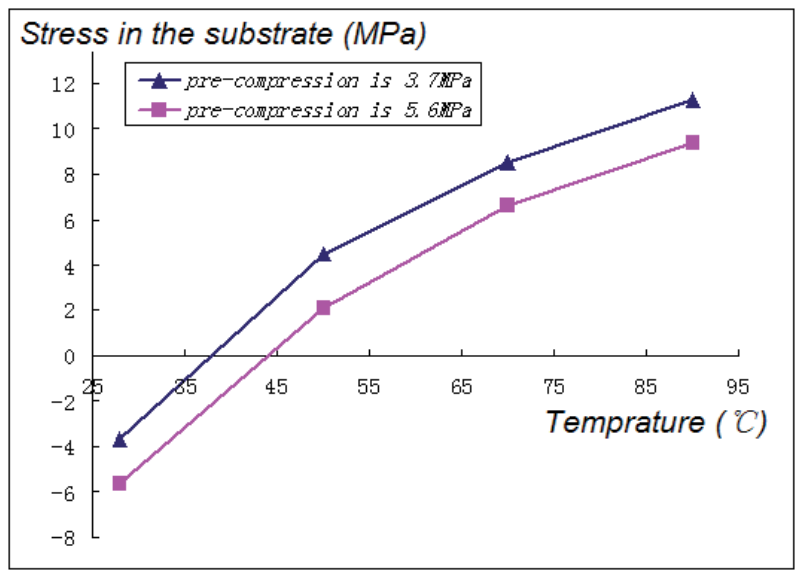

Fig. 6 The average stress change at the observation points as the temperature changing,

When pre-compression are separately 3.7 MPa and 5.6MPa.

As the temperature increasing, average stress of the observed location continued to increase gradually from the compressive stress into tensile stress. This shows that the distribution of stress in the in the substrate is an important factor in changes of the topology of thin film buckling.

\section{Conclusions}

The model of film deposited on substrate is widely used in technology, which will endure mechanical loads besides thermal load for its generating quality of heat. The qualitative analysis of the model of film deposited on substrate which is under mechanical and thermal loading is made for 
limitation of method of technology and theory. It is found that the size and number of the delamination and buckling of the film are depended upon the pre-fixed mechanical loading, and the distribution of stress in the in the substrate is an important factor in changes of the topology of thin film buckling.

\section{Acknowledgement}

We acknowledge the support from the NSFC (10732080), DF (20060056012) and TJNF (06YFJZJC00700).

\section{References}

1. Cotterell B., Chen Z., Int J of Fract. 2000, 104: 169 179

2. Hutchinson J. W., Suo Z., Adv Appl Mech. 1992; 29: 63 191

3. Hutchinson J. W., He M. Y., J. Mech Phys Solids. 2000; 48: 709 734

4. Moon M. W., Jensen H. M., J.Mech Phys Solids. 2002, 50: $2355 \sim 2377$

5. Lee A., Clemens B. M., Acta Mater. 2004; 52: 2081 2093

6. Eymery J. P., Denanot M. F., Surf Coat Tech. 1996, 80: 251 254

7. Moon M. W., Lee K. R., Acta Mater. 2004, 52: 3151 3159

8. S. B. Wang, H. K. Jia, X. C. Ren, L. A. Li. Proc. SPIE. 2008, 7375: 73753b 